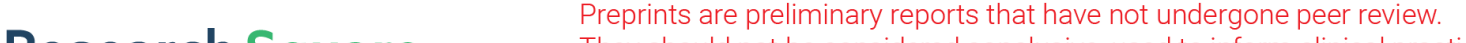 \\ They should not be considered conclusive, used to inform clinical practice, \\ or referenced by the media as validated information. \\ The Effect of Different Surface Plasmon Polaritons Shapes on Thin Film Solar Cell Efficiency
}

\section{Khalil ElKhamisy ( $\nabla$ k.mahmoud@sha.edu.eg )}

El-Shorouk Academy: Shorouk Academy https://orcid.org/0000-0002-3423-5356

\section{Salah Elagooz}

El-Shorouk Academy: Shorouk Academy

\section{El-Sayed El-Rabaie}

Menoufia University

\section{Hamdy Abdelhamid}

Zewail City of Science and Technology - 6th of October City Campus

\section{Research Article}

Keywords: Thin film Si solar cell, surface plasmon polaritons, efficiency improvement

Posted Date: April 30th, 2021

DOI: https://doi.org/10.21203/rs.3.rs-457172/v1

License: (c) (1) This work is licensed under a Creative Commons Attribution 4.0 International License. Read Full License

Version of Record: A version of this preprint was published at Journal of Computational Electronics on July 7th, 2021. See the published version at https://doi.org/10.1007/s10825-021-01729-0. 


\title{
The Effect of Different Surface Plasmon Polaritons Shapes on Thin Film Solar Cell Efficiency
}

\author{
Khalil ElKhamisy ${ }^{1}$, Salah Elagooz ${ }^{1}$, El-Sayed El-Rabaie ${ }^{4}$, and Hamdy Abdelhamid ${ }^{2,3}$ \\ ${ }^{1}$ Department of Communication and Computer Engineering, Higher Institute of Engineering (HIE), El- \\ Shorouk City 11837, Egypt \\ ${ }^{2}$ Center of Nano-Electronics and Devices (CND), Zewail City of Science and Technology, 6th October \\ City, Egypt \\ ${ }^{3}$ Faculty of Engineering and Information Technology, Electrical Engineering Department, Ajman \\ University, Ajman, United Arab Emirates \\ ${ }^{4}$ Department of Electronics and Communications Faculty of Electronic Engineering, \\ Menoufia University, Egypt
}

(Corresponding Author: Khalil ElKhamisy, mail: k.mahmoud@sha.edu.eg )

Keywords Thin film Si solar cell, surface plasmon polaritons, efficiency improvement

Abstract - Thin film Si solar cell and surface plasmon polaritons (SPPs) effects on solar cell efficiency, series resistance and shunt resistance are studied and analyzed in this work. The different surface plasmon polaritons (SPPs) shapes and their effects on the optical, electrical properties and therefore on the efficiency of thin film solar cell are studied in this work. This study is introduced using 3D numerical simulation results. The semiconductor and electromagnetic models are incorporated for studying the electrical and optical behaviors of the thin film solar cells, respectively. A $\mathbf{1 4 . 7 6 \%}$ efficiency is obtained for triangle' SPPs of about $1.07 \%$ of efficiency improvement compared to solar cell of SPPs free. The solar cell electrical parameters also are extracted in this work based on a single diode equivalent model. The series resistance is enhanced for solar cells of equilateral triangle SPP by $3 \%$ compared to the non-applied SPPs.

\section{INTRODUCTION}

Photovoltaic is the cleanest way to get an electric energy by converting the solar energy into electrical energy [1].

The demands for increasing the photovoltaic efficiency is the main challenge that facing the solar cell usages spreading and mass production respecting the cost. This due to the material and process expenses, definitely for bulk silicon based solar cells [2].

The thin film solar cells have reduced the cost by replacing the bulk substrate by thin film layers instead of the bulk one. The main issue of using the thin film technology is reducing the absorbed energy level down to the semiconductor material energy gap. Thin-film silicon solar cells have different advantages but they still want to achieve moral efficiency and improve production methods. Thin-film silicon solar cells technology has clashed with recent crystalline silicon cells, utilizing a limited amount of active materials and record low-thermal growth. Although crystalline silicone solar cells grow rapidly in thin-film [3-6].

Plasmonics paid great attention to these challenges by using metals to harness light during a deep-subwavelength calculation; plasmonics have accepted a substantial 
reduction in photonic system size [7-10]. Plasmonic gratings-periodic metallic structures on surfaces used to enhance plasmonic fields. The grating increases plasmonic pattern propagation along the grating. Beyond the waveguide attitude, an apparent advantage of this mode of plasmonic improvement is that it does not require unconnected couplings. The optical fiber geometry can accompany these geometries for the detector. Overall, plasmonic grating-type detectors are better suited to obtain the signal directly from free space than the wave-guide-form due to wave-guide coupling failures [11-13].

Surface plasmon polaritons (SPPs), elevating excitations along metal-dielectric boundaries arising from the combination of light and cooperative fluctuations of uncontrolled electrons, have attracted considerable attention in the research regions of physics , chemistry, biology and material science, especially (lower diffraction threshold) due to their exclusive properties [14-17].

The manufacturing techniques and applications of SPPs in solar cells are growing rapidly in recent years. A new crystalline silicon solar cell, with Si nano-cone arrays on the top and $\mathrm{Al}$ nano-hemisphere arrays on the bottom side. The $3 \mathrm{D}$ numerical simulations using COMSOL Multiphysics systematically studied the capacity to trap light. The nano-cone arrays gain light fastening by adding gradient refractive shift incident light is indexed and integrated into optical modes. The metallic nanohemisphere arrays affect light harvesting by polaritons of surface plasmon (SPPs) and the scattering effect [18]. A new standard $\alpha$-Si thin solar cell film. We build Ag nanograting to spread more light incident. It also exhibits in the meantime a silver/S- $\alpha$ plasmonic interface enhancement. Energy caught The design will increase short circuit current density to $23.74 \mathrm{~mA} / \mathrm{cm}^{2}$ on both front anti-reflective coating and the back metal reflector. From $300 \mathrm{~nm}$ to $950 \mathrm{~nm}$, over the solar spectrum. This style we also find can maintain a high density of the short circuit current within a wide incidence angle variation [19]. COMSOL Multiphysics, a package for finite elemental analysis, is used to research the effect of nanogratic structures on a-Si thin film solar cell. The method gives the requisite geometry simulation economic efficiency and convenience. Nevertheless, it can be said that various tests were conducted before actual calculations were carried out to authenticate the proper functioning of the model using Fresnel equations well known in literature. The effects on light trapping (absorption) are regulated by seven gate structures with variable slit widths (slits). The construction of the grating becomes simpler to produce Optical antennas and separated ring similar to nanoparticles Resonators and so forth [20]. Silver nanoparticles combined with a thinfilm solar cell (TFSC) silicon. It is made of $\mathrm{TiO} 2$ pyramids in which the antireflective layer is made of anti-reflective layers. The goal of this structure is to allow sunlight to penetrate the cell at any angle with the minimum reflexion. and 300-1100 nm wavelength absorption. Silizium is the absorbing layer, and the molecular bonds break down and discharge many electrons by their high absorption rate when sunlight enters this layer. Silver spherical nanoparticles are positioned in this layer to increase solar energy absorption through localized plasmon resonances on the surface [21]. Plasmonic grating on the surface is very useful for controlling the absorbed light, manipulating and detecting light with specific polarisation. The plasmonic grating contributes to increased absorption and shows excellent efficiency in reducing losses and allowing light power propagation in optoelectronic devices [22].

A Multiphysics toolbox is used in this study to precisely model various shapes of SPPs on thin film solar cells. The contributions of this study are modeling five different shapes of SPPs, studying the effect of each shape on the efficiency of thin film solar cells, and extracting the equivalent circuit parameters for each one. 
The remainder of the paper structured as follows, structure of models and parameters of used models in Section 2. Optical and electrical model in section 3. The effects of the simulation given in Sec. 4. Finally, our observations of our work are summarized in Section 5.

\section{MODEL STRUCTURE AND PARAMETERS}

The structure of the modeled solar cell as shown in Fig. 1, consisting of four stacked layers: a protective $\mathrm{SiO} 2$ layer at the top and the a-Si layer absorbers as a P-I-N structure, followed by another $\mathrm{SiO} 2$ layer at the rear, the whole layer structure constructed on top of the reflector behind. The intrinsic thickness of the amorphous silicon is $260 \mathrm{~nm}$. In this paper, $40 \mathrm{~nm}$ thick layers $\mathrm{N}+$ and $\mathrm{P}+$ and $500 \mathrm{~nm}$ thick back reflector. The doping profile of $\mathrm{P}+$ and $\mathrm{N}+$ layers is $1 \times 10^{19}\left[\mathrm{~cm}^{-3}\right]$ and $1 \times$ $10^{19}\left[\mathrm{~cm}^{-3}\right]$ respectively.

Fig. 1 (b, c, d, e and f) shows the different SPPs shapes using gold simulated in this work to study the optical and electrical behaviors of the solar cell. Fig. 2 presents the doping profile of the used P-I-N layers.

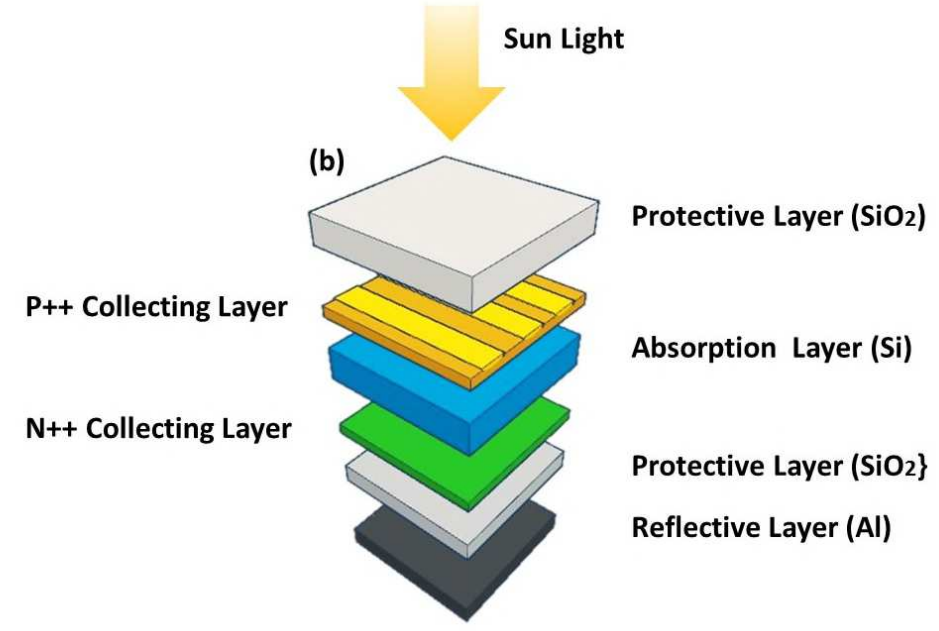

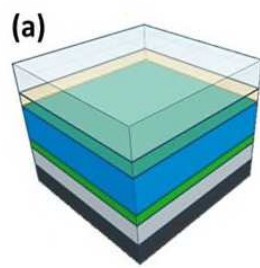

(d)

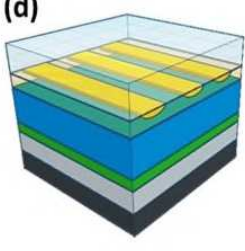

(b)

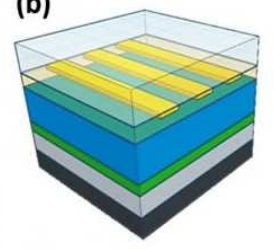

(e)

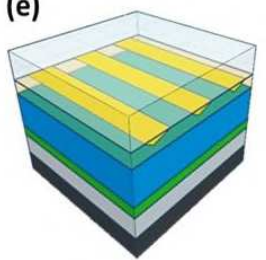

(c)
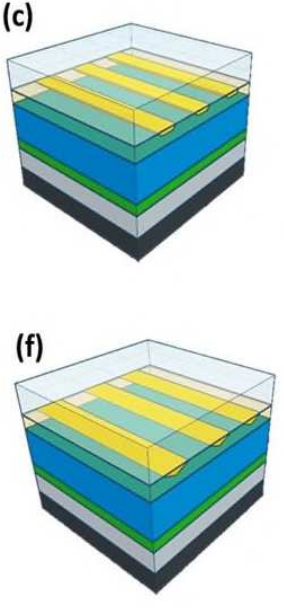

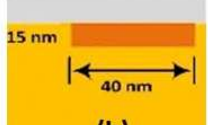

(b)
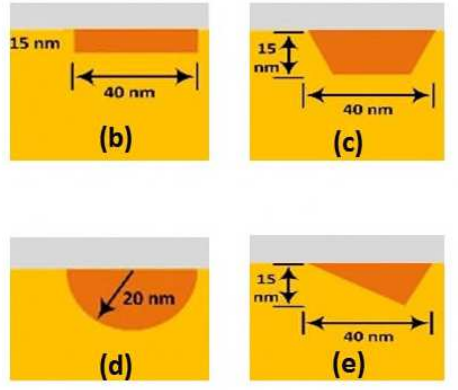

(e)

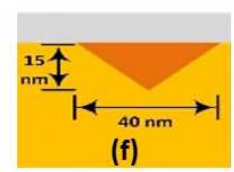


Fig. 1. Structure of the P-I-N device of different types of SPPs, (a) without SPP [23], (b) square SPP, (c) trapezoidal SPP, (d) half circle SPP, (e) scalene triangle SPP, and (f) equilateral triangle SPP.

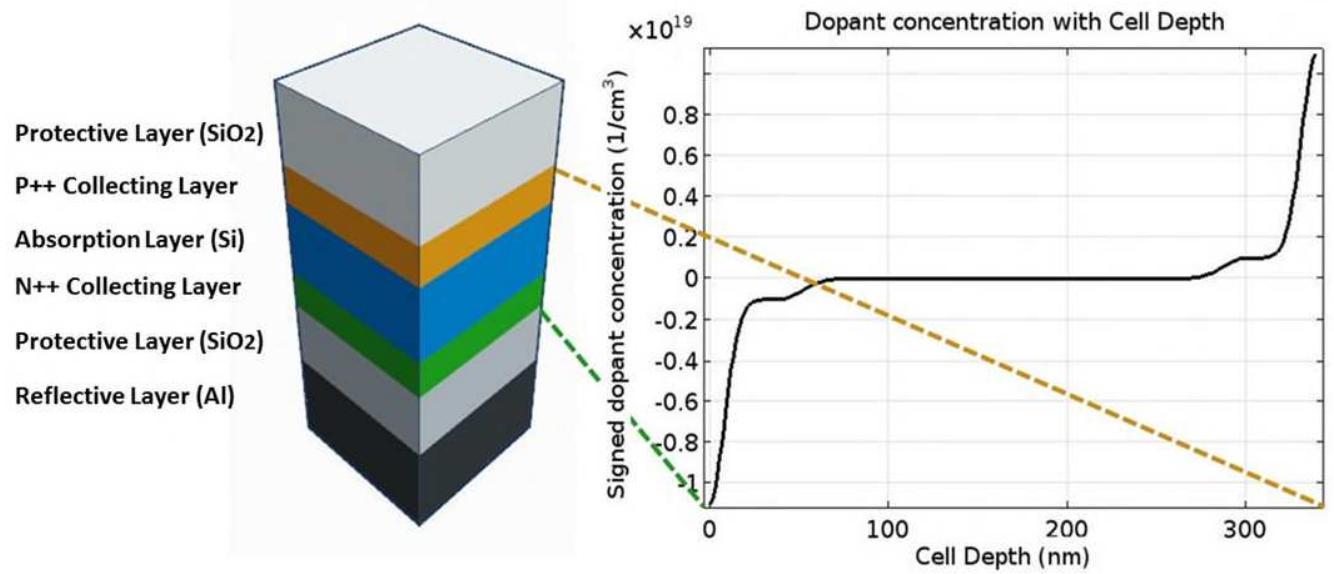

Fig. 2. The doping profile in P-I-N device model simulation for P-N device.

\section{Mathematical model}

\subsection{The Excitation of SPP}

SPPs will excite along with photons and electrons. The excitation with electrons is produced in the metallic mass as a result of exciting electrons. The light energy is distributed in plasma form [24] at the same time as the electrons are dispersed.

Aimed to rouse an SPP at a photon, they each have to have the same frequency and momentum. The free-space photon meant to be defined for such a frequency is stronger than the SPP momentum, since both have dissimilar dispersion relations. Such a disparity in momentum causes the photon in free space to pair directly from air to the SPP. The SPP can't produce energy in the dielectric as a free-space photon atop a smooth out metal surface, aimed for the same purpose. Such a misalignment refers to the lack of transmission that occurs through absolute inner reflection.

\subsection{The Fields and dispersion relation in SPP}

From Maxwell's equations, the SPP characteristics can be obtained. Here, $\mathrm{Z}=0$ at metal - dielectric interface, $\mathrm{Z}<0$ through the metal and $\mathrm{Z}>0$ through dielectric. As a function of position $(\mathrm{x}, \mathrm{y}, \mathrm{z})$ and time $\mathrm{t}$ the electric and magnetic fields can be written as $[25,26]$,

$$
\begin{gathered}
E_{x, n}(x, y, z, t)=E_{0} e^{i k_{x} x+i k_{z, n}|z|-i w t} \\
E_{z, n}(x, y, z, t)= \pm E_{0} \frac{k_{z}}{k_{z, n}} e^{i k_{z} x+i k_{z, n}|z|-i w t} \\
H_{y, n}(x, y, z, t)=H_{0} e^{i k_{x} x+i k_{z, n}|z|-i w t}
\end{gathered}
$$


Where $\mathbf{n}$ refers to physical material ( 1 for $\mathrm{z}<0$ metal or 2 for $\mathrm{z}>0$ dielectric). The angular frequency of the waves is $\boldsymbol{\omega} . \pm$ is + to the metallic, - to the dielectric. $\boldsymbol{E}_{\boldsymbol{x}}, \boldsymbol{E}_{\boldsymbol{z}}$ for the electric field vector are the x- and z- parts, $\boldsymbol{H}_{\boldsymbol{y}}$ the magnetic field vector is the y- part, in addition to the other parts $\left(\boldsymbol{E}_{\boldsymbol{x}}, \boldsymbol{E}_{\boldsymbol{z}}, \boldsymbol{H}_{\boldsymbol{y}}\right)$ are zero. The SPPs are TM (transverse magnetic) waves forever. $\boldsymbol{K}$ is the wave vector; it is a compound vector, and when a lossless SPP occurs, it transforms the $\boldsymbol{x}$ parts into real and the $z$ elements into imaginary elements-the wave fluctuates along the $\boldsymbol{x}$ path and exponentially in the $\boldsymbol{z}$ path. $\boldsymbol{k}_{\boldsymbol{x}}$ is often the same as intended for the content when $\boldsymbol{k}_{\boldsymbol{z}, \mathbf{1}}$ is usually different from $\boldsymbol{k}_{\boldsymbol{z}, \mathbf{2}}$, where,

- $\frac{H_{0}}{E_{0}}=-\frac{\varepsilon_{1} w}{k_{z, 1} C}$ or $\frac{H_{0}}{E_{0}}=-\frac{\varepsilon_{2} w}{k_{z, 2} C}$. where $\varepsilon_{1}$ is permittivity of the metallic.

In subsequent equations, a wave of this structure fulfills Maxwell's equations in single state, or

And

$$
\frac{k_{z, 1}}{\varepsilon_{1}}+\frac{k_{z, 2}}{\varepsilon_{2}}=0
$$

$$
K^{2}{ }_{x}+K_{z n}^{2}=\varepsilon_{n}\left(\frac{w}{c}\right)^{2} \quad n=1,2
$$

Eq. (4) and Eq. (5) can be solved to obtain the dispersion relationship for a wave spreading at the top surface as,

$$
k_{\mathrm{z}}=\frac{w}{C}\left(\frac{\varepsilon_{1} \varepsilon_{2}}{\varepsilon_{1}+\varepsilon_{2}}\right)^{1 / 2}
$$

The metal dielectric formula is obtained within the free electron model of the electron stream, which avoids reduction [27], or

$$
\varepsilon(w)=1-\frac{w_{p}^{2}}{w^{2}}
$$

Where the frequency of plasma on SI units can be written as,

$$
W_{P}=\sqrt{\frac{n e^{2}}{\varepsilon_{0} m^{*}}}
$$

Here $\boldsymbol{n}$ is the electron density, $\boldsymbol{e}$ is the electron's charge, $\boldsymbol{m}^{*}$ is the electron 's effective mass and $\boldsymbol{\varepsilon}_{\mathbf{0}}$ is the free-space permittivity. The SPP acts as a photon at low $\boldsymbol{k}$, but the dispersion relationship bows and reaches an asymptotic boundary called the "surface plasma frequency" as it rises.

Similar to metal-SPP, it is sufficient to assume that the surface wave often induces metal-dielectric multilayer pairing between the electromagnetic field and electronic fluctuation [28]. This wave is confined to the metamaterial boundary and spreads alongside the interface. The electromagnetic field normally decreases exponentially according to the limited energy near the boundary and will not spread within the substantial. As shown in Fig.3, the thickness of the Plasmon blank substratum depends on the imaginary portion of the dielectric constant in thin films and its position is strengthened by the dint of the dielectric layer's wideness. From Fig. 3, the dispersion relationship varies and the angle is transferred as the thin dielectric layer is adsorbed to the top of the thin metal film sheet. This resonance angle swing is proportional to the optical thickness, which is influenced by the difference between each of the refractive indices and the depth of the thin film. 


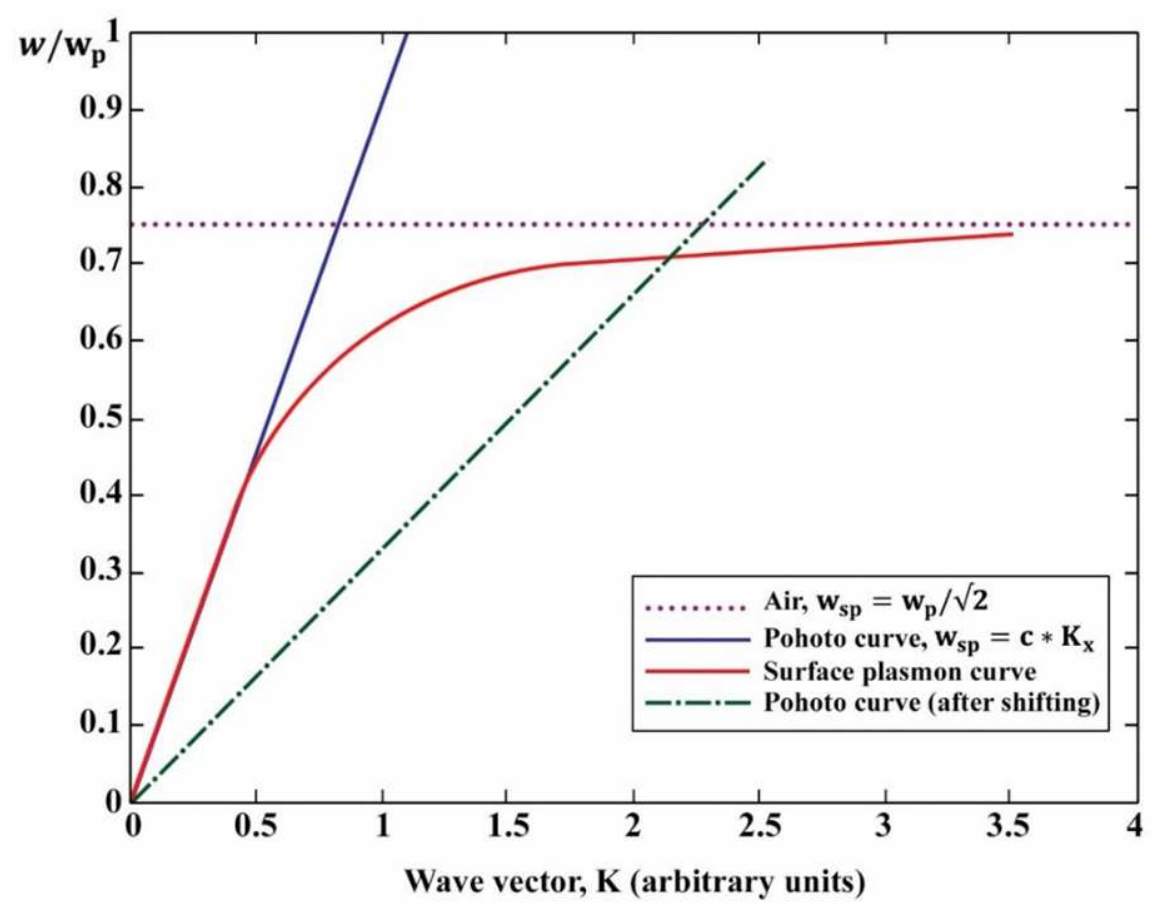

Fig. 3 The dispersion relative to the photon through surface plasmon, as k grows the dispersion curve leans down and reaches the asymptotic limit [27].

\subsection{The Propagation length and skin depth in SPP}

So an SPP stretches the top lengthwise, because of the absorption it drops energy toward the metallic. The surface plasmon concentration falls off the square of the electric field, so at a distance $\mathrm{x}$, the intensity decreased by the factor of $\boldsymbol{e x p}\left\{-2 \boldsymbol{k}_{x}^{=}\right\}$(x not subscript, as I believe). The length of propagation is expressed as the expanse to decline by a factor of $1 / e$ to the SPP power. This condition is fulfilled to a degree [29]

$$
\boldsymbol{L}=\frac{1}{2 \boldsymbol{k}_{x}^{2}}
$$

Similarly, the electric field evanescently decreases making it a corner to the top of the metallic. The SPP diffusion depth within this metal is typically calculated through the formulation of the skin depth at small frequencies. The field within the dielectric will slowly decrease further beyond. The measurements of decline within the metal and dielectric medium recorded in place of follows [30]

$$
Z_{i}=\frac{\lambda}{2 \pi}\left(\frac{\left|\varepsilon^{\prime}{ }_{1}+\varepsilon_{2}\right|}{\varepsilon^{2} i}\right)^{1 / 2}
$$

Where ' $i$ ' define propagation intermediate. SPPs are very sensitive to small skin depth disorders and as a result, SPPs are also used to examine top inhomogeneity. 


\subsection{Five-Parameter Model (single diode model)}

The single-diode standard, as shown in the Fig. 4 consist of four main parameters as follows: the existing photovoltaic action source $\boldsymbol{I}_{\boldsymbol{p}}$, and the diode itself. The ideal recombinant current of electrons and holes in cell-side diffusion and recombination (Shockley diffusion theory), $\boldsymbol{R}_{\boldsymbol{s} e}$ and $\boldsymbol{R}_{\boldsymbol{s} h}$ accounting for various causes of loss and no ideals.

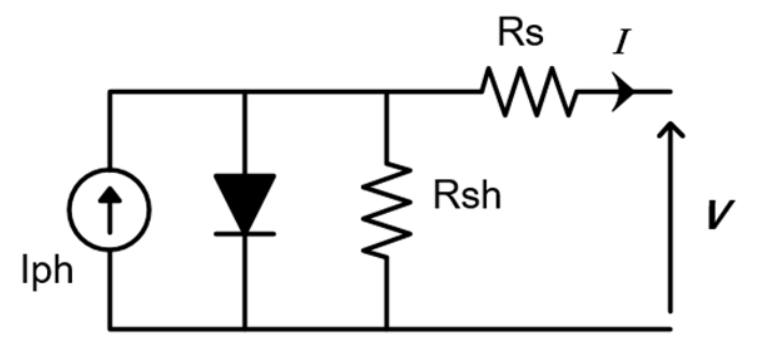

Fig. 4 Equivalent circuit of a solar cell [31].

Where:

$$
\begin{aligned}
& R_{s}=\frac{V_{o c}-V_{m}}{10 \cdot I_{m}} \\
& R_{s h}=\frac{10 \cdot V_{m}}{I_{s c}-I_{m}} \\
& K_{1}=\frac{R_{s h}-R_{s}}{R_{s}} \cdot\left[\frac{I_{m} \cdot\left(R_{s h}+R_{S}\right)-V_{m}}{V_{m}-I_{m} \cdot R_{s}}\right] \\
& n=\frac{V_{m}+R_{s} \cdot\left(I_{m}-I_{s c}\right)}{V_{t} \cdot \ln (K 1)} \\
& I_{0}=\frac{R_{s} \cdot n \cdot V_{t}}{R_{s h} \cdot\left(R_{s h}-R_{S}\right)} \cdot \exp \left[\frac{-I_{s c} \cdot R_{s}}{n \cdot V_{t}}\right] \\
& I_{p h}=I_{s c}+I_{0} \cdot\left[\exp \frac{I_{s c} \cdot R_{s}}{n \cdot V_{t}}-1\right]+\frac{I_{s c} \cdot R_{S}}{R_{s h}}
\end{aligned}
$$

Where Io is the diode's reverse saturation current, $\boldsymbol{n}$ is a non-dimensional constant called the ideality factor that defines the diode's deviation from the Shockley diffusion principle, $\boldsymbol{V}_{\boldsymbol{o c}}$ and $\boldsymbol{I}_{\boldsymbol{s} \boldsymbol{c}}$ are respectively the open circuit voltage and the short circuit current [31]. Ultimately, the thermal stress is $\boldsymbol{V}_{\boldsymbol{t}}$.

The main electrical parameters for the solar cell such as solar cell output, open circuit voltage, and short circuit current $[32,33]$ can be extracted from our simulation-based model.

$$
\text { Solar Cell Efficiency }(\%)=\frac{J_{s c} V_{o c} F F}{P_{\text {in }}}
$$

FF is the filling factor, which can derived from $\mathrm{J}-\mathrm{V}$ curve of the device as,

$$
F F=\frac{I_{m} V_{m}}{I_{s c} V_{o c}}
$$




\section{P-I-N DEVICE SIMULATION RESULTS}

\subsection{Optical parameters simulation Results}

Fig. 5 shows the results for simulating the propagation of the electrical field in the $x$ and y direction of certain structures. In the cross-sectional area Fig. 5a, the effect of electric field strength on the silicone substrate for a typical PIN-device without the SPP is indicated. For SPP-free thin film solar cell. Fig. 5f the light intensity across the SPP is visible along the aperture, when the electric field intensity is maximized, because the incident light helps electrons swap their unique features like plasmon polaritons with the help of the Au SPP Fig. $5 \mathrm{f}$ The thin film solar cell with equilateral SPP triangularity. In Fig. 5a, 5f at the right up it can be observed from the electric field intensity figure that there is an enhancement using Au SPP.
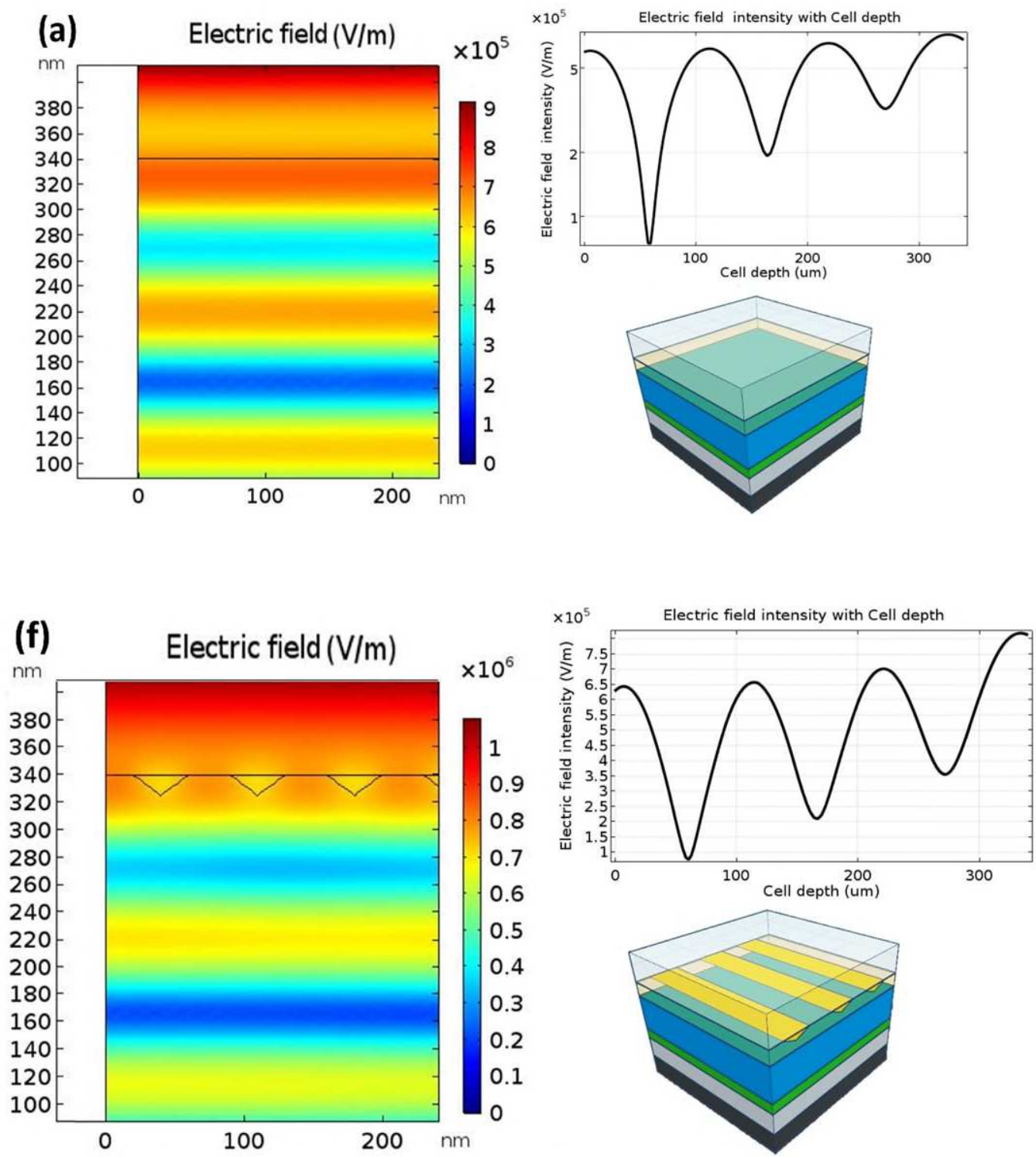

Fig. 5. The electric field in different shapes of SPPs, (a) without, and (f) equilateral triangle SPP. 


\subsection{Electrical parameters simulation Results}

The results of the simulation for this P-I - N device model, as shown in Figure 6 and Table 1, as well as power curves for the presented models with different forms of SPPs, provide complete details about the output data presented. In the case of an efficiency without an SPP of $13.69 \%$, a little more of the efficiency between the previous two models for a rectangle SPP was $14.47 \%$. However, the efficiency for trapezoidal PSP was $14.44 \%$, with a further small improvement of around $0.45 \%$ for half-circle SPP. The best result in this analysis was $14.76 \%$ for the equilateral SPP triangle, with an average increase of $1.07 \%$ between the first and last iterations.

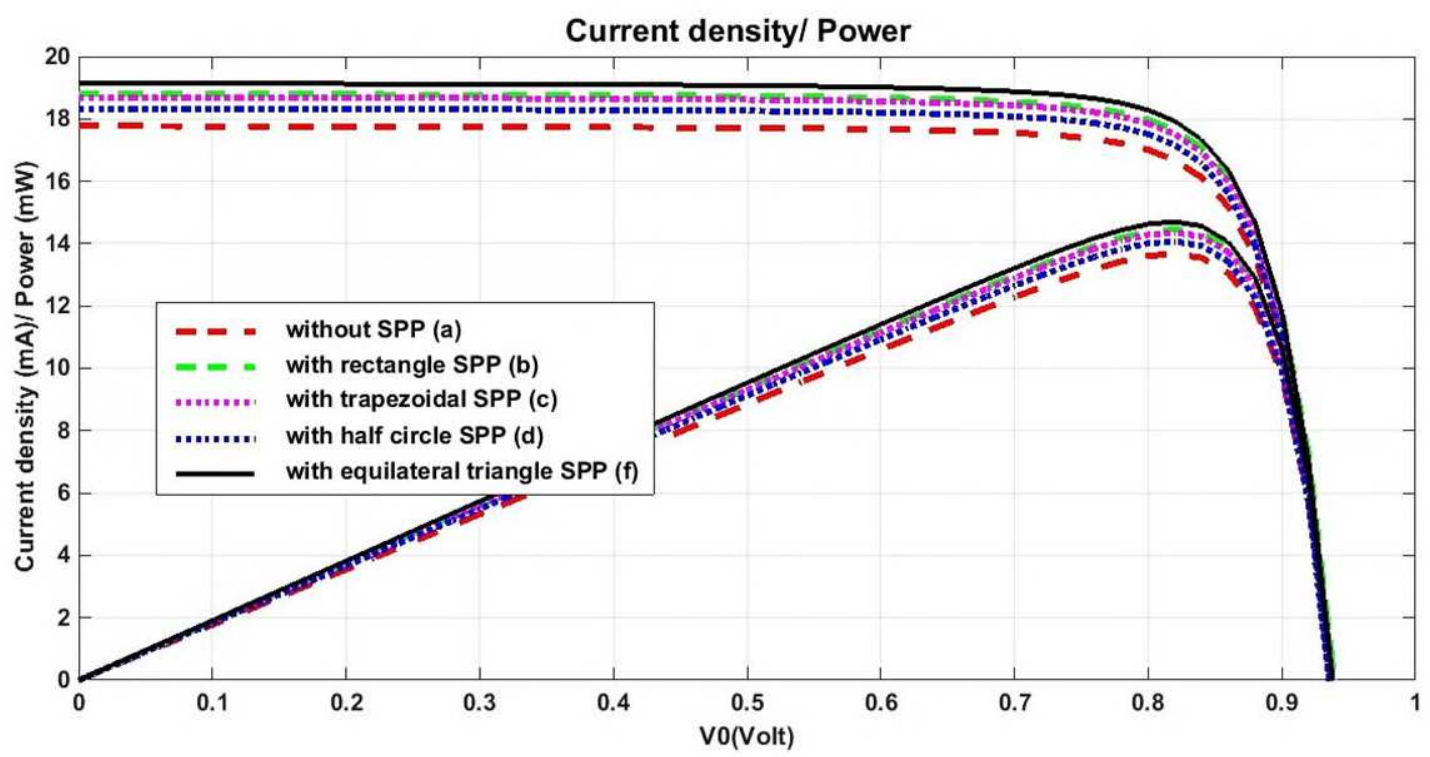

Fig. 6. J-V / P-V curve in P-I-N device model with different types of surface grating, (a) without SPP, (b) square SPP, (c) trapezoidal SPP, and (d) half circle SPP, and (f) equilateral triangle $\mathbf{S P P}$.

The Top SPP triangle uses the scatter effects to allow a lighter penetration into the solar cell, thereby maximizing overall absorption and electrical power over the other grid, as illustrated in Table 1. The rear reflector of the thin-film solar cell reflects and disperses light that not absorbed in the first phase through the membranes. The shapes and the sizing of the SPP are based on the cell's diffraction of the incident solar spectrum, determining the amount of power produced by the cell. When the back reflactor substrate is added at the bottom of the structure, the unabsorbed photons are also redirected to a P-I - N junction, optimizing light entanglement and increasing solar efficiency.We look at the spectrum, because it has an effect of 0.843 in the equilateral triangle SPP on total electricity generated from the solar cell. For whole cases, the solar cell 's maximum absorbed power is increased by $14,65[\mathrm{~mW}]$ regardless of the position of proposed equilateral SPP triangle. 
Table 1. The result values of P-I-N device.

\begin{tabular}{|c|c|c|c|c|c|c|c|}
\hline $\begin{array}{c}\text { Parameter } \\
\text { Name }\end{array}$ & $\begin{array}{c}\text { Without } \\
\text { SPP }\end{array}$ & $\begin{array}{c}\text { Rectangle } \\
\text { SPP }\end{array}$ & $\begin{array}{c}\text { Trapezoidal } \\
\text { SPP }\end{array}$ & $\begin{array}{c}\text { Half-Circle } \\
\text { SPP }\end{array}$ & $\begin{array}{c}\text { scalene } \\
\text { Triangle SPP }\end{array}$ & $\begin{array}{c}\text { Equilateral } \\
\text { Triangle } \\
\text { SPP }\end{array}$ & $\begin{array}{c}\text { Equilateral } \\
\text { Triangle } \\
\text { grating [6] }\end{array}$ \\
\hline $\mathrm{P}_{\text {in }}\left[\mathrm{W} / \mathrm{m}^{2}\right]$ & 1000 & 1000 & 1000 & 1000 & 1000 & 1000 & 1000 \\
\hline $\mathrm{P}_{\max }[\mathrm{mW}]$ & 13.7 & 14.5 & 14.35 & 14.1 & 14.6 & 14.7 & 12.5 \\
\hline $\begin{array}{c}\mathrm{J}_{\mathrm{sc}} \\
{\left[\mathrm{mA} / \mathrm{cm}^{2}\right]}\end{array}$ & 17.75 & 18.8 & 18.7 & 18.3 & 19.1 & 19.2 & 13 \\
\hline $\mathrm{V}_{\mathrm{oc}}[\mathrm{V}]$ & 0.93 & 0.933 & 0.932 & 0.931 & 0.934 & 0.935 & 1.08 \\
\hline $\begin{array}{c}\mathrm{J}_{\mathrm{m}} \\
{\left[\mathrm{mA} / \mathrm{cm}{ }^{2}\right]}\end{array}$ & 16.7 & 17.65 & 17.6 & 17.15 & 17.9 & 18 & 12.5 \\
\hline $\mathrm{V}_{\mathrm{m}}[\mathrm{V}]$ & 0.82 & 0.82 & 0.82 & 0.82 & 0.82 & 0.82 & 0.98 \\
\hline $\mathrm{FF}$ & 0.829 & 0.825 & 0.828 & 0.823 & 0.82 & 0.822 & 0.872 \\
\hline $\begin{array}{c}\mathrm{Efficiency} \\
(\%)\end{array}$ & 13.69 & 14.47 & 14.43 & 14.06 & 14.67 & 14.76 & 12.25 \\
\hline $\mathrm{n}$ & 3.34 & 3.38 & 3.34 & 3.42 & 3.43 & 3.40 & 3.56 \\
\hline $\mathrm{I}_{0}[\mathrm{~A}]$ & $1.2 \mathrm{E}-06$ & $9.23 \mathrm{E}-08$ & $8.32 \mathrm{E}-08$ & $1.03 \mathrm{E}-07$ & $1.10 \mathrm{E}-07$ & $1.00 \mathrm{E}-07$ & $1.562 \mathrm{E}-8$ \\
\hline $\mathrm{I}_{\mathrm{ph}}[\mathrm{A}]$ & 0.0177 & 0.0188 & 0.0187 & 0.0183 & 0.0191 & 0.0192 & 0.013 \\
\hline $\mathrm{R}_{\mathrm{s}}[\Omega]$ & 0.658 & 0.640 & 0.636 & 0.674 & 0.636 & 0.638 & 0.800 \\
\hline $\mathrm{R}_{\mathrm{sh}}[\Omega]$ & 780 & 713 & 745 & 638 & 656 & 683 & 1960 \\
\hline
\end{tabular}

At the rest of Table 1 we summarized the calculated values for the PV electrical parameters $\left(I_{0}, I_{p h}, \boldsymbol{R}_{s}\right.$. and $\left.\boldsymbol{R}_{\boldsymbol{s} h}\right)$. It is noticed that the electrical parameters are enhanced definitely for equilateral triangle SPP based technique. The last column in Table 1 shows the results of Ref. [6] by compare it with the simulated model at the case of equilateral triangle SPP the observation is more enhancement in Efficiency and $\boldsymbol{R}_{\boldsymbol{s}}$ about $2.51 \%$ and $25 \%$ reapectivly.

\section{CONCLUSIONS}

In this paper, both electrical and optical characteristics of the thin film solar cells are examined. Various SPPs shaped in order to increase the absorption rate and related dimensions are studied. The SPPs generally improved photovoltaic efficiency besides the maximum output power compared to the thin film solar cells. A significant improvement are achieved for triangle shaped SPPs based thin film solar cells. The best results of this analysis for the SPP triangle were $14.76 \%$, the total performance change between the first and last versions was $1.7 \%$. In the SPP equilateral triangle, we also consider the spectrum because it affects total power generated by the solar cell and a fill factor of 0.822 . Finally we observed that the location of equilateral triangle SPP the maximum power absorbed by the solar cell is enhanced by $14.7[\mathrm{~mW}]$. The $\mathrm{R}_{\mathrm{s}}$ value improved by about $3 \%$ for the equilateral SPP triangle. 
6.

Author Contribution All the authors have contributed equally.

Funding No funding was received.

Data Availability Data related to this article are available from the corresponding author upon reasonable request.

\section{Declarations}

Consent to Participate All the authors agreed to involve in this research work.

Consent for Publication All the authors have given permission to publish the results.

Conflict of Interest The authors declare that they have no conflict of interest.

\section{REFERENCES}

[1] S.R. Wenham, M.A. Green, M.E. Watt, R. Corkish, A. Sproul, Applied Photovoltaics, Routledge, 2007.

[2] A.L. Fahrenbruch, and R.H. Bube, "Fundamentals of Solar Cells," Academic Press Inc., New York. 1983.

[3] A. Shah," Thin-Film Silicon Solar Cells", EPFL Press, Lausanne, Switzerland, 2010.

[4] S. A. Maier, M. L. Brongersma, P. G. Kik, S. Meltzer, A. A. G.Requicha, and H.A. Atwater, "Plasmonics - A route to nanoscale optical devices," Advanced Materials, Article vol. 13, no. 19, Oct 2001.

[5] Khalil M. ElKhamisy, S. El-Rabaie, Salah S. Elagooz, and Hamdy Abd Elhamid " The effect of different surface grating shapes on thin film solar cell efficiency" 2019 International Conference on Innovative Trends in Computer Engineering, Aswan, pp. 297-300, 2-4 February 2019.

[6] Bedir Yousif, Mohy Eldin A. Abo-Elsoud and Hagar Marouf, " Triangle grating for enhancement the efficiency in thin film photovoltaic solar cells", Optical and Quantum Electronics,Springer,Vol. 51 ,pp. 276(4-11) ,August 2019.

[7] H. A. Atwater and A. Polman, "Plasmonics for improved photovoltaic devices," Nature Materials, Review vol. 9, no. 3, pp. 205-213, Mar 2010.

[8] J. A. Schuller, E. S. Barnard, W. S. Cai, Y. C. Jun, J. S. White, and M.L.Brongersma, "Plasmonics for extreme light concentration and manipulation," Nature Materials, Review vol. 9, no. 3, pp. 193-204, Mar 2010.

[9] D. K. Gramotnev and S. I. Bozhevolnyi, "Plasmonics beyond the diffraction limit," Nature Photonics, Review vol. 4, no. 2, pp. 83-91, Feb 2010.

[10] M. Kauranen and A. V. Zayats, "Nonlinear plasmonics," Nature Photonics, Review vol. 6, no. 11, pp. 737-748, Nov 2012.

[11] J. Leuthold et al., "Plasmonic Communications: Light on a Wire," vol. 24, no. $5,2013$.

[12] C. Haffner et al., "All-plasmonic Mach-Zehnder modulator enabling optical high-speed communication at the microscale," Nature Photonics, Article vol.9, no. 8, Aug 2015. 
[13] M. L. Brongersma, "Plasmonic Photodetectors, Photovoltaics, and HotElectron Devices," Proceedings of the IEEE, Article vol. 104, no. 12, pp. 2349-2361, Dec 2016.

[14] S. A. Maier, Plasmonics: Fundamentals and Applications. NewYork, USA: Springer, 2007.

[15] W. L. Barnes, A. Dereux, and T. W. Ebbesen, "Surface plasmon subwavelength optics," Nature, vol. 424, pp. 824, Aug. 2003.

[16] D. K. Gramotnev, and S. I. Bozhevolnyi, "Plasmonics beyond the diffraction limit," Nat. Photon., vol. 4, pp. 83, Jan. 2010.

[17] J. A. Schuller et al., "Plasmonics for extreme light concentration and manipulation," Nat. Materials, vol. 9, pp. 193, Feb. 2010.

[18] Zhaopeng Xu, Huichao Huangfu, Xiaowei Li, Huiling Qiao, Wanchun Guo, Jingwei Guo, and Haiyan Wang," Role of nanocone and nanohemisphere arrays in improving light trapping of thin film solar cells" Optics Communications, vol. 377, pp. 104-109, 2016.

[19] Jing Hao, Zhongyuan Yu, Y umin Liu, Han Ye, and Jinqiannan Zhang," Light Trapping Nanostructure Design for Absorption Enhancement in Amorphous Silicon Thin Film Solar Cell " Optical Nanostructures and Advanced Materials for Photovoltaics, Boulder, Colorado United States 69 November 2017.

[20] Tahir Iqbal1, Mohsin Ijaz, Muhammad Javaid, Muhammad Rafique, Khalid Nadeem Riaz, Muhammad Bilal Tahir, Ghulam Nabi1, Muhammad Abrar,and Sumera Afsheen," An Optimal Au Grating Structure for Light Absorption in Amorphous Silicon Thin Film Solar Cell " Plasmonics, vol. 14, pp. 147-154, 2018.

[21] Afsaneh Asgariyan Tabrizi, and Ali Pahlavan," Efficiency improvement of a silicon-based thin-film solar cell using plasmonic silver nanoparticles and an antireflective layer " Optics Communications, vol. 454, pp. 124437, 2020.

[22] Bedir Yousif, Mohy Eldin A. Abo-Elsoud, and Hagar Marouf," HighPerformance Enhancement of a GaAs Photodetector Using a Plasmonic Grating" Plasmonics, 2020.

[23] Ahmadreza Ghahremani \& Aly E. Fathy, "A three-dimensional multiphysics modeling of thin-film amorphous silicon solar cells", Energy Science and Engineering, 3(6), 2015, pp.520-534.

[24] M. J. Lockyear, A. P. Hibbins, and J. R. Sambles, "Microwave surfaceplasmon-like modes on thin metamaterials," Phys. Rev. Lett., Vol. 102, No. 7, pp. 073901, Feb. 2009.

[25] Zeng, Shuwen; Yu, Xia; Law, Wing-Cheung; Zhang, Yating; Hu, Rui; Dinh, Xuan-Quyen; Ho, Ho-Pui; Yong, Ken-Tye (2013). "Size dependence of Au NP-enhanced surface plasmon resonance based on differential phase measurement". Sensors and Actuators B: Chemical. 176: 1128-1133.

[26] Raether, Heinz (1988). Surface Plasmons on Smooth and Rough Surfaces and on Gratings. Springer Tracts in Modern Physics 111. New York: Springer-Verlag. ISBN 978-3540173632. 
[27] Cottam, Michael G. (1989). Introduction to Surface and Superlattice Excitations. New York: Cambridge University Press. ISBN 9780750305884

[28] Kittel, Charles (1996). Introduction to Solid State Physics (8th ed.). Hoboken, NJ: John Wiley \& Sons. ISBN 978-0-471-41526-8

[29] J. Dostalek, J. Ctyroky, J. Homola, E. Brynda, M. Skalsky, P. Nekvindova, J. Spirkova, J. Skvor, and J. Schrofel, "Surface plasmon resonance biosensor based on integrated optical waveguide," Sensors and Actuators B: Chemical, vol. 76, pp. 8-12, 2001..

[30] Homola, Jirí (2006). Surface Plasmon Resonance Based Sensors. Springer Series on Chemical Sensors and Biosensors, 4. Berlin: Springer-Verlag. ISBN 978-3-540-33918-2.

[31] Hamdy Abdelhamid, Ahmed Edris, Amr Helmy, and Yehea Ismail," Fast and accurate PV model for SPICE simulation", Journal of Computational Electronics,(18)2,2019,pp. 260-270.

[32] Ahmadreza Ghahremani \& Aly E. Fathy, "A three-dimensional multiphysics modeling of thin-film amorphous silicon solar cells", Energy Science and Engineering, 3(6), 2015, pp.520-534.

[33] J. J. Quinn, "Solid state physics: principles and modern applications", Springer-Verlag, Berlin Heidelberg, 2009. 


\section{Figures}

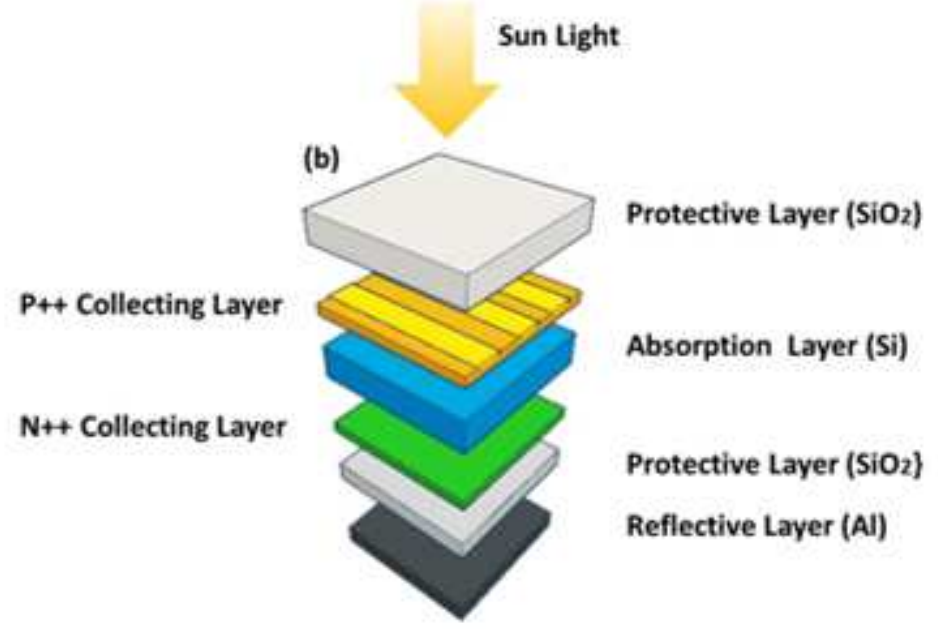

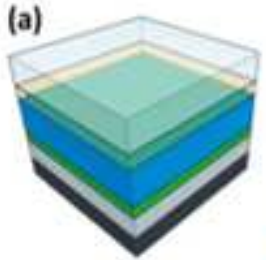

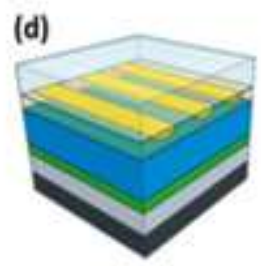

(b)

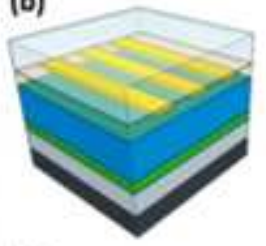

(e)

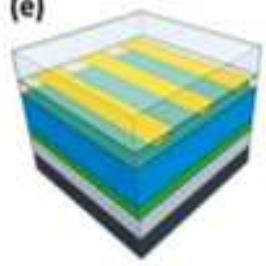

(c)

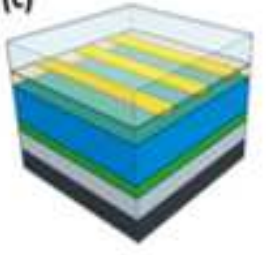

(f)

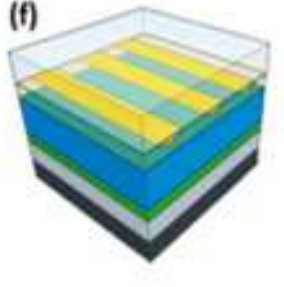

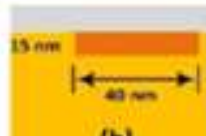

(b)
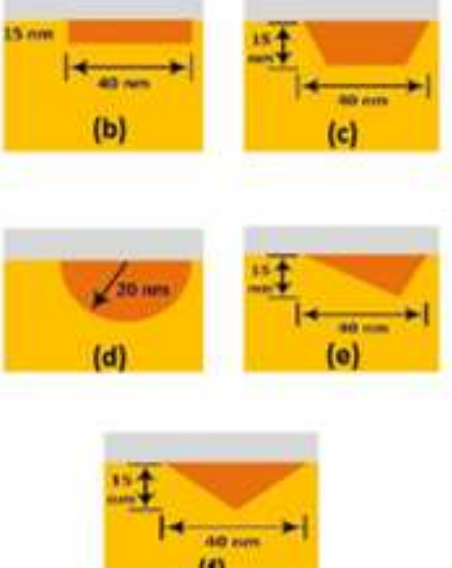

(f)

\section{Figure 1}

Structure of the P-I-N device of different types of SPPs, (a) without SPP [23], (b) square SPP, (c) trapezoidal SPP, (d) half circle SPP, (e) scalene triangle SPP, and (f) equilateral triangle SPP. 


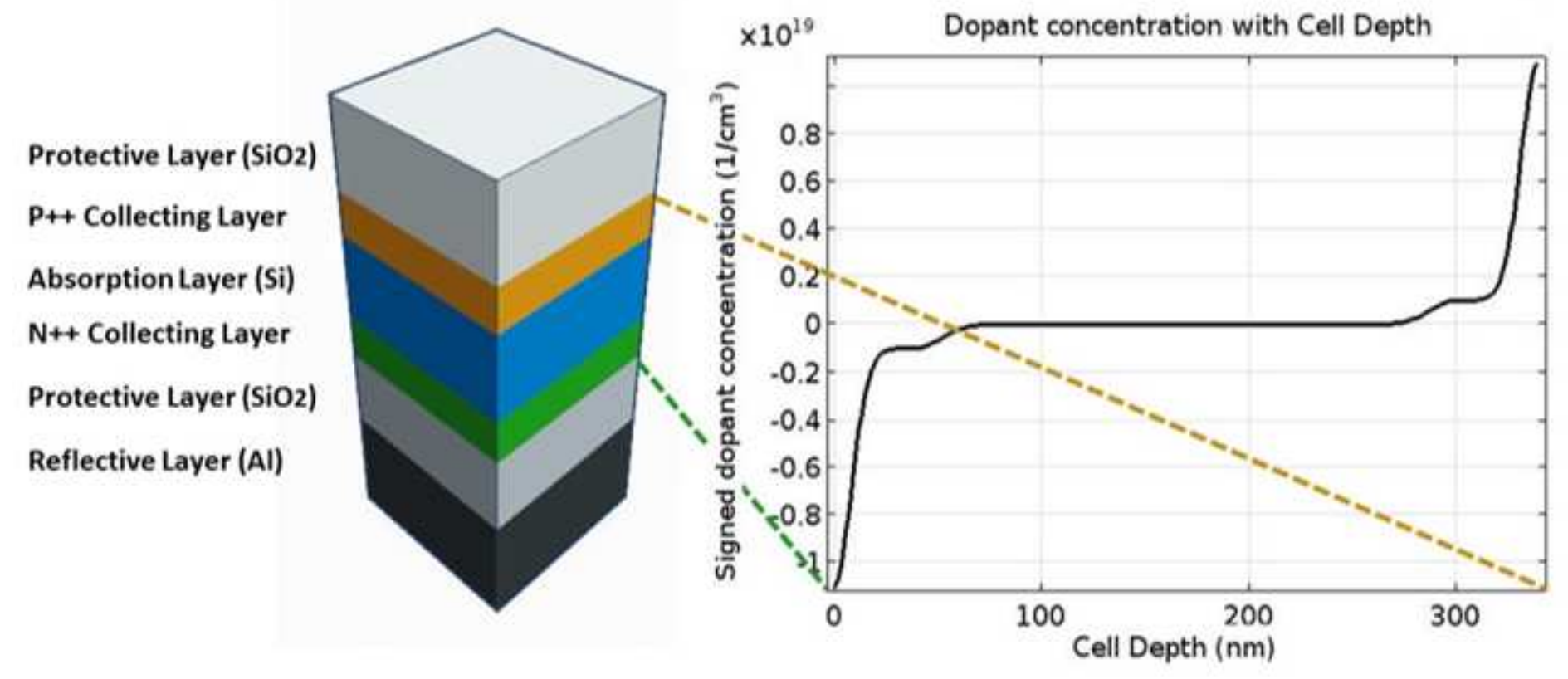

Figure 2

The doping profile in P-I-N device model simulation for P-N device. 


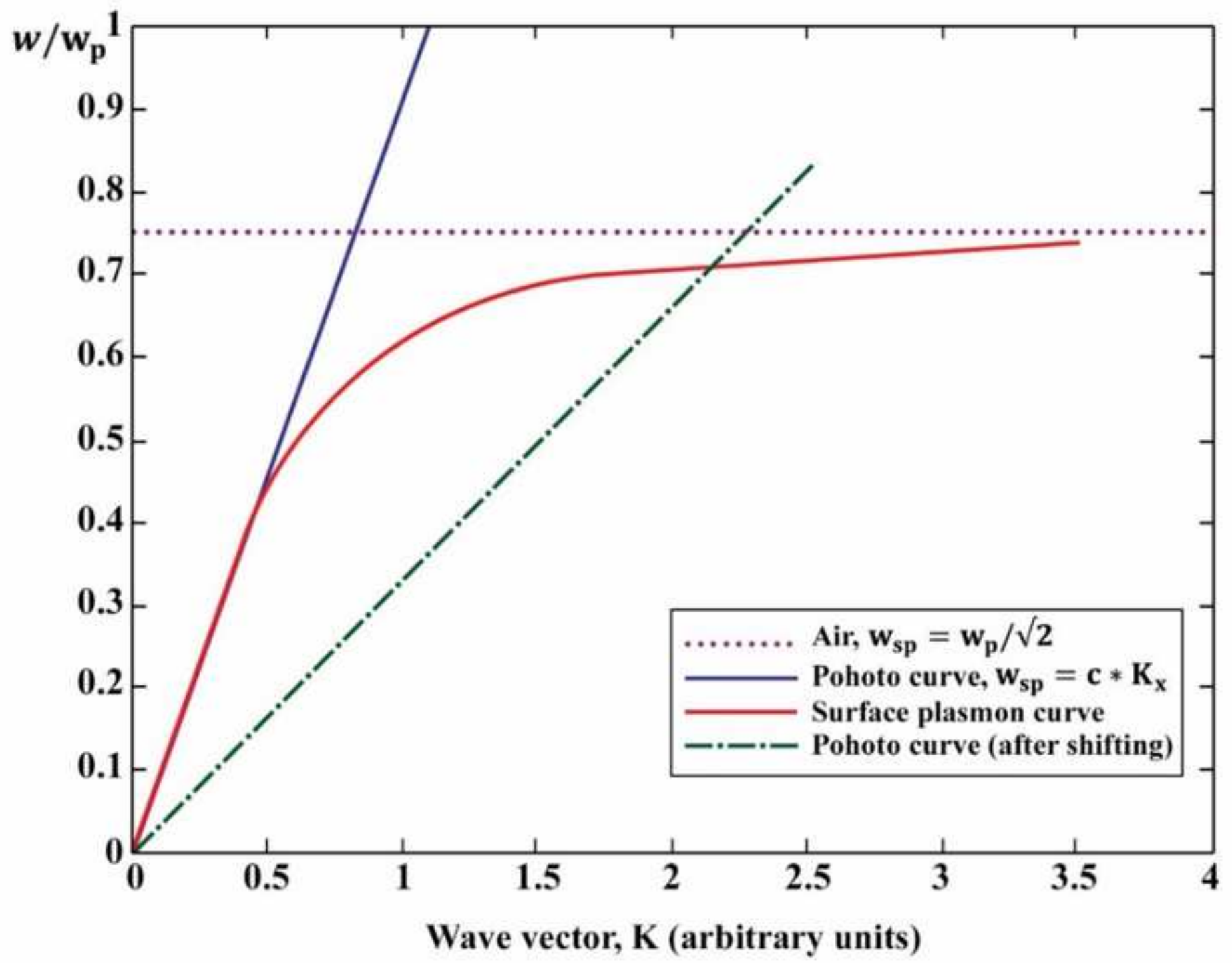

Figure 3

The dispersion relative to the photon through surface plasmon, as $\mathrm{k}$ grows the dispersion curve leans down and reaches the asymptotic limit [27].

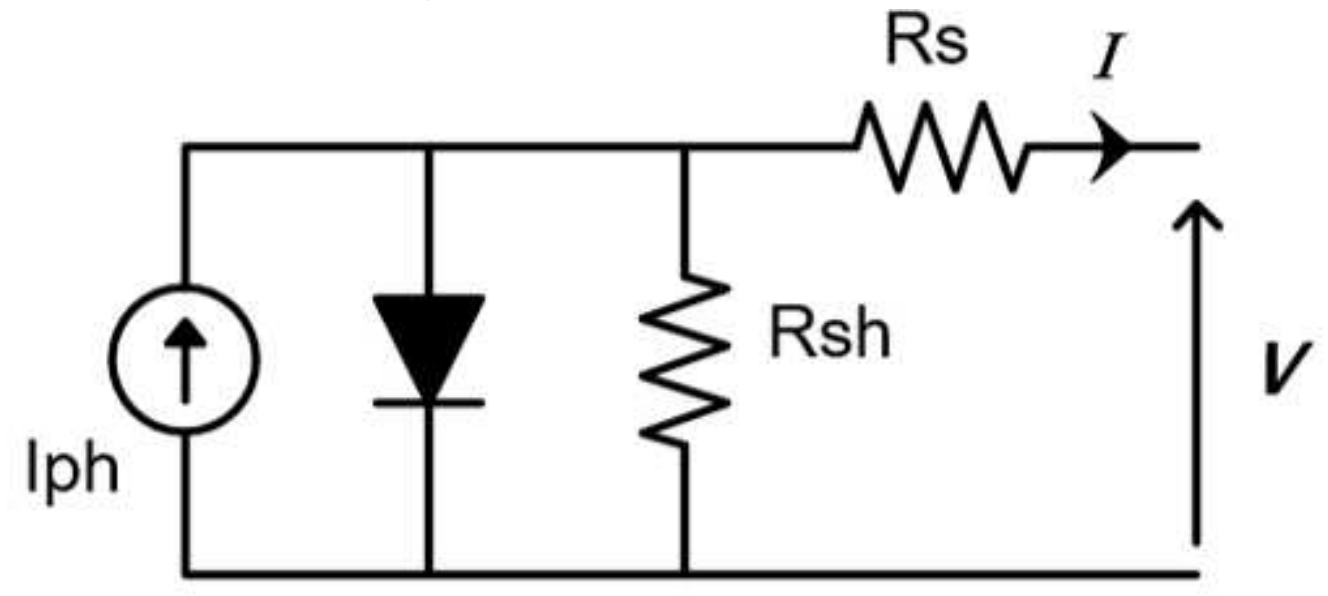

Figure 4 
Equivalent circuit of a solar cell [31].
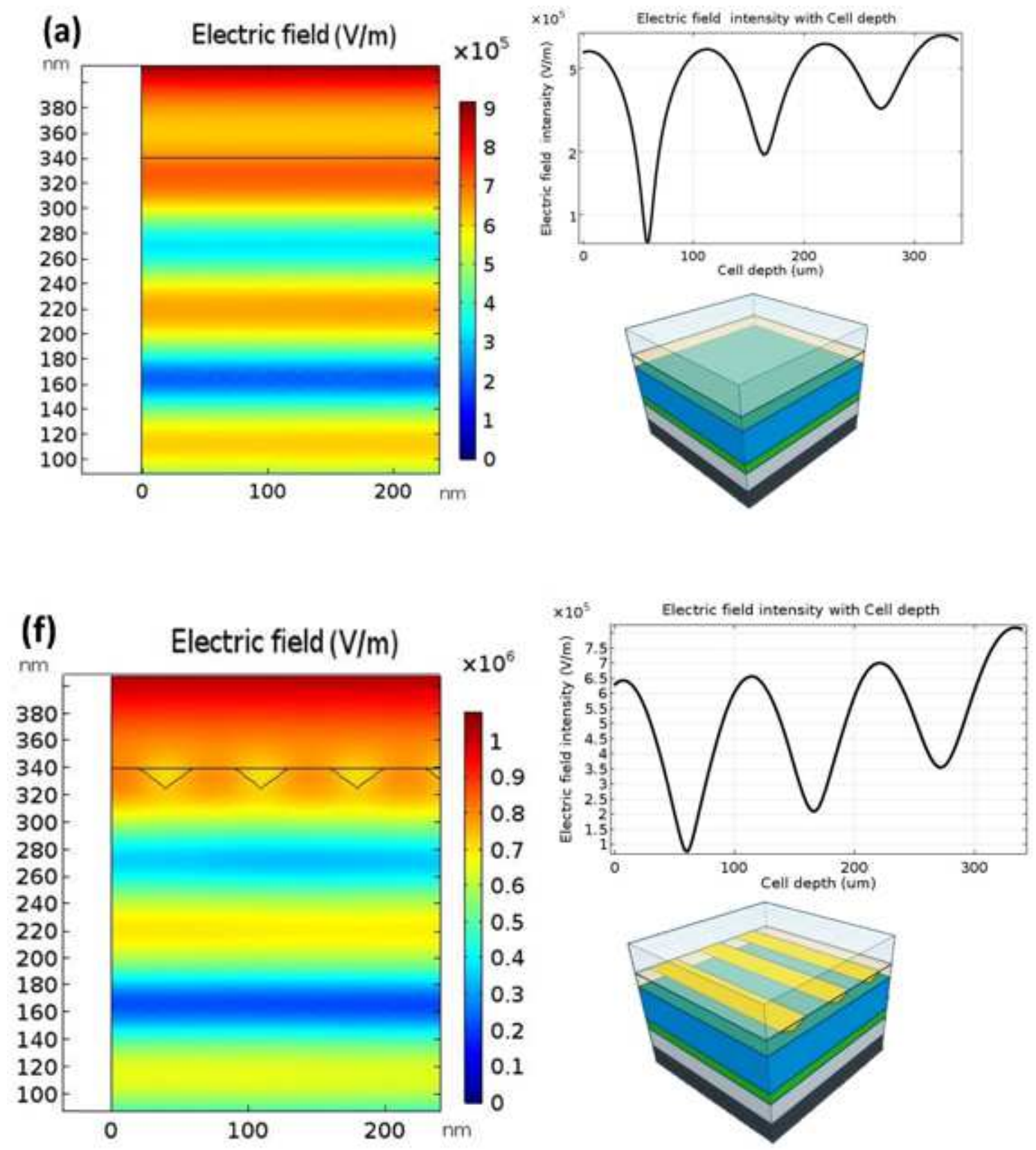

Figure 5

The electric field in different shapes of SPPs, (a) without, and (f) equilateral triangle SPP. 


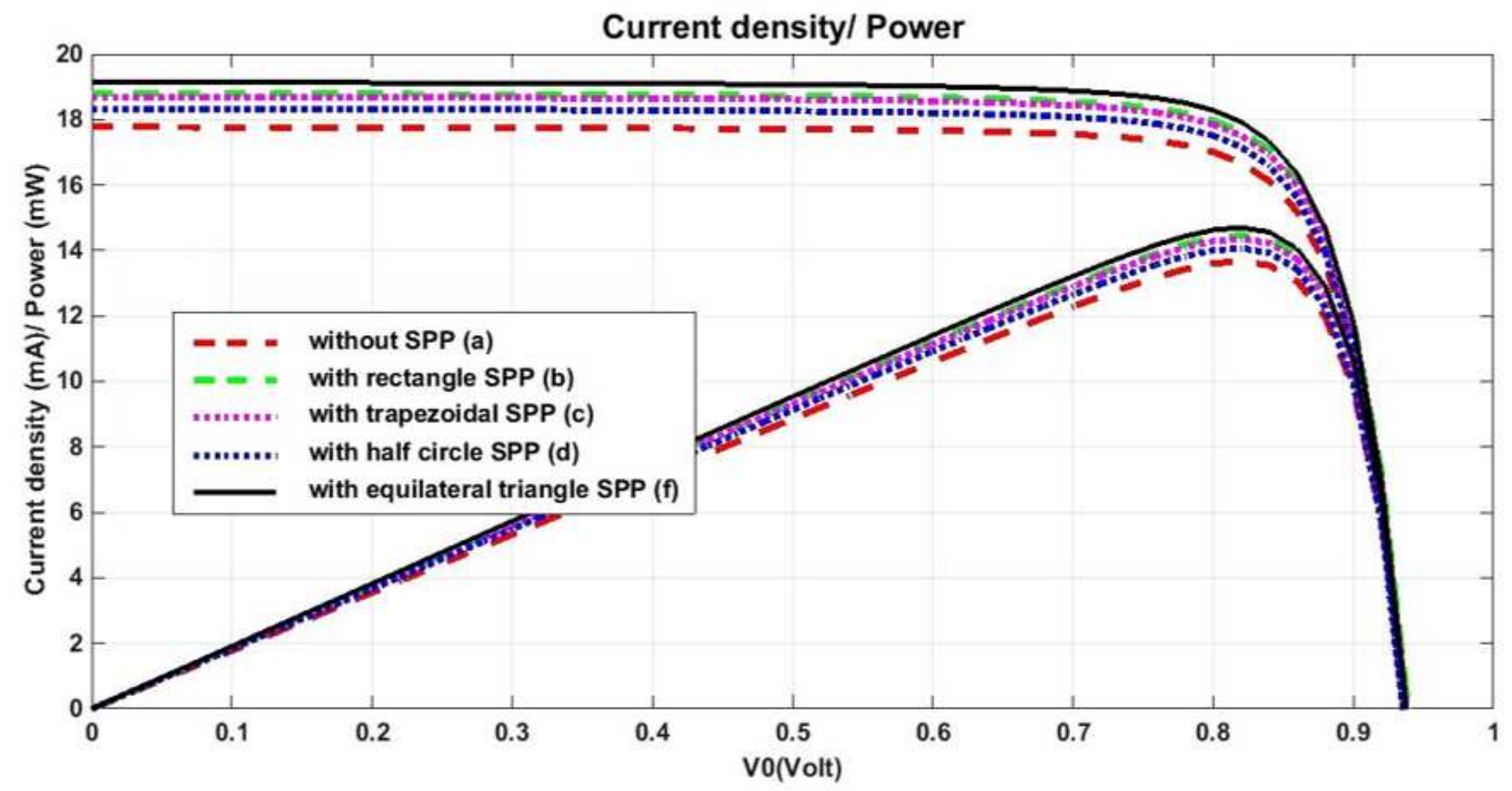

Figure 6

J-V / P-V curve in P-I-N device model with different types of surface grating, (a) without SPP, (b) square SPP, (c) trapezoidal SPP, and (d) half circle SPP, and (f) equilateral triangle SPP. 